\title{
EFFETS QUADRUPOLAIRES \\ DANS LA RÉSONANCE MAGNÉTIQUE NUCLÉAIRE DES ALLIAGES DILUÉS
}

\author{
Par A. BLANDIN ET J. FRIEDEL, \\ Service de Physique des Solides, Faculté des Sciences. - Orsay.
}

\begin{abstract}
Résumé. - Les fortes variations d'intensité de la résonance magnétique nucléaire dans les alliages dilués sont dues à la variation de densité électronique $\Delta \rho$ créée à grande distance par les . impuretés dissoutes. Aux noyaux distants de $r$ d'une impureté, $\Delta \rho$ a la forme :

$$
\text { Cte } \times \cos (2 k r+\varphi) / r^{3}
$$

et donne lieu à un gradient de champ ayant la même variation avec la distance et une constante dépendant fortement du caractère $s, p$ ou $d$ des fonctions d'onde au niveau de Fermi du métal pur. Les différents cas (alliages d'Al, Cu) étudiés expérimentalement sont discutés sur la base de cette théorie. Des conclusions analogues ont été obtenues simultanément par Kohn et Vosko [18].

Abstract. - The strong intensity changes in the nuclear magnetic resonance of dilute alloys are the consequence of the electronic density oscillations $\Delta \rho$ which are created at large distances by the dissolved impurities.

For a nucleus at a distance $r$ from an impurity, $\Delta \rho$ is proportional to the function $\cos \left(2 k_{\mathrm{F}} r+\varphi\right) / r^{3}$ and gives field gradients of the same asymptotic form, multiplied by a constant depending strongly on the $s, p$ or $d$ character of the Bloch wave functions at the Fermi level of the pure metal. The experimental results for $\mathrm{Cu}$ or $\mathrm{Al}$ base alloys are discussed. Analogous conclusions have been simultaneously obtained by Kohn and Vosko [18].
\end{abstract}

I. Introduction. - Les résultats expérimentaux de Bloembergen et Rowland [1] et de Rowland [2] sur les alliages à base de cuivre et d'aluminium indiquent de très forts abaissements de l'intensité des raies de résonance magnétique nucléaire du cuivre ou de l'aluminium pour de très faibles concentrations d'impuretés. Ces résultats impliquent l'existence de forts gradients de champ électrique au voisinage des impuretés suffisants pour rendre inobservables les résonances des voisins (jusqu'aux $7^{\text {es }}$ voisins dans certains cas).

L'origine de ces gradients de champ a été assez mal comprise jusqu'à présent. L'objet de cet article est de montrer qu'ils ne peuvent être créés par des effets purement élastiques [3], mais qu'ils sont la conséquence des oscillations de charge électronique créées par les impuretés : ces oscillations étudiées par Friedel [4] ont permis d'expliquer les variations du déplacement de Knight dans les alliages [5] et les propriétés magnétiques des alliages à impuretés de transition [6]. Elles donnent des gradients de champ à grande distance de la forme

$$
A \cos \left(2 k_{\mathrm{F}} r+\varphi\right) / r^{3}
$$

où $A$ et $\varphi$ sont des constantes. $A$ dépend fortement de la nature des fonctions d'onde au niveau de Fermi.

Dans le paragraphe 2, les principales conclusions expérimentales sont sommairement rappelées. Le paragraphe 3 traite des effets élastiques, le paragraphe 4, de la diffusion des électrons de Bloch par une impureté, dans une méthode de Wigner-Seitz étendue. On en déduit les variations de densité électronique et les gradients de champ (\$5). Ceux-. ci sont évalués au paragraphe 6.. Enfin le paragraphe 7 rassemble les résultats obtenus et les compare aux résultats expérimentaux.

II. Les résultats expérimentaux. - Dans les alliages de cuivre et d'aluminium [1, 2], les raies de résonance nucléaire ne sont pratiquement pas déplacées et gardent la même largeur que dans les métaux purs ; mais leur intensité décroît très rapidement pour de très petites concentrations $(<0,1 \%)$ d'impuretés. Ceci s'explique dans un modèle du " tout ou rien » imaginé par Bloembergen et Rowland [1,2,3]. L'impureté crée des gradients de champ $q$ : pour tous les noyaux soumis à un gradient de champ supérieur à une valeur $q_{0}$ déterminée de sorte que le déplacement du $1^{\text {er }}$ ordre dans la fréquence de résonance des raies statellites $(3 / 2 \leftarrow \rightarrow 1 / 2 ;-3 / 2 \leftarrow \rightarrow-1 / 2$ pour un spin nucléaire $I=3 / 2$ ) soit de l'ordre de la largeur de raie, les raies satellites ont des fréquences de résonance trop différentes et les atomes ne participent plus à l'intensité totale. Si $q<q_{0}$, au contraire, il n'y a aucun effet. L'intensité $I$ de la résonance correspondant aux raies satellites doit alors suivre. aux petites concentrations $c$, une loi de la forme:

$$
\log I / I_{0}=n \log (1-c) \simeq-n c,
$$

$n$ étant le nombre d'atomes entourant une impureté et soumis à un gradient de champ supérieur à $q_{0}$. Cette loi est bien vérifiée et donne des valeurs de $n$ de 98 et 130 pour les alliages $\mathbf{A l} \mathbf{Z n}$ et $\mathbf{A l} \mathbf{~ M g}$ pour une valeur de $q_{0}$ égale à $6.10^{21} \mathrm{~cm}^{-3}$.

Dans le cas des alliages $\mathrm{Cu} \mathrm{Zn}$, c'est un .effet du 
second ordre qui est observé. Le modèle de Bloembergen et Rowland doit être meilleur dans ce cas, car les gradients décroissent vite sur les premiers voisins et les déplacements de fréquence dépendent des carrés des gradients de champ. Il donne $n=18$ pour $q_{0}=1,5.10^{23} \mathrm{~cm}^{-3}$. Les valeurs de $q_{0}$ sont calculées en prenant de même que Rowland [3] la valeur critique du déplacement de fréquence égale à 5 kilocycles.

III. Rôle des déformations élastiques. - Bloembergen [3] a étudié l'effet des déformations élastiques : celles-ci produisent un gradient de champ à la distance $r$

$$
q=\lambda \frac{6 \varepsilon}{r^{3}} \quad \text { où } \quad \varepsilon=\frac{a-r_{\mathrm{A}}}{r_{\mathrm{A}}}
$$

est le changement relatif du rayon d'équilibre $a$ de l'impureté par rapport au rayon $r_{\mathrm{A}}$ de la matrice. $\lambda$ est un facteur numérique lié à la matrice qui tient compte de la distorsion des couches atomiques internes dans le réseau perturbé. Pour expliquer les résultats expérimentaux, il est nécessaire de prendre des valeurs de $\lambda$ égales à 30 et 60 pour l'aluminium et le cuivre. Mais, en traitant comme une déformation élastique la présence d'une impureté $B$ dans la matrice $A$, on trouve pour le rayon d'équilibre $a$ [7] :

$$
\frac{a-r_{\mathrm{A}}}{r_{\mathrm{B}}-r_{\mathrm{A}}}=x \frac{\chi_{\mathrm{A}}}{\chi_{\mathrm{B}}}
$$

où $\chi_{\mathrm{A}}$ et $\chi_{\mathrm{B}}$ sont les compressibilités de $\mathrm{A}$ et $\mathrm{B}$ et $x=1+\nu / 2(1-2 v), \nu$ étant le module de Poisson de la matrice A. La différence $r_{\mathrm{B}}-r_{\mathrm{A}}$ est donnée par la variation de paramètre cristallin avec la concentration mesurée aux rayons $X$, par exemple [8]. Dans le cas des alliages $\mathbf{A l ~} \mathrm{Zn}$ et $\mathbf{A l ~ M g}$, alors que $r_{\mathrm{A}}=1,58 \AA$ on trouve pour $a$ respectivement : $1,56 \AA$ et $1,70 \AA$. Les effets sont très différents alors que les nombres $n$ tirés des mesures de N. M. R. sont du même ordre : 98 et 130. Clairement, les gradients de champ créés dans le cristal par la distorsion élastique due aux atomes B ne permettent pas de comprendre ces résultats.

De récentes expériences d'Averbuch et al. [9] ont confirmé ce point de vue : en effectuant la résonance magnétique nucléaire sur du cuivre écroui et en mesurant aux rayons $\mathrm{X}$ les distorsions du cristal, on peut obtenir une mesure expérimentale du facteur $\lambda$; on trouve pour le cuivre, comme ordre de grandeur, $\lambda \simeq 1$, au lieu de $\lambda=60$ proposé par Bloembergen.

L'ensemble de ces considérations permet de conclure qu'il faut chercher ailleurs l'origine des importants gradients de champ que l'on observe dans les alliages. L'explication donnée ici est que les gradients de champ sont créés par le changement de répartition électronique autour des impuretés.
IV. Structure électronique d'une impureté dans l'approximation de Wigner-Seitz. - La variation de densité électronique créée en un point autour d'une impureté et le gradient de champ que l'on en déduit dépendent fortement de la position du point dans la cellule de Wigner-Seitz auquel il appartient. Il faut donc, ainsi que cela sera vérifié dans les conclusions, traiter la perturbation due à l'impureté avec précaution, c'est-à-dire en dehors de l'approximation des électrons libres $\left({ }^{\mathbf{1}}\right)$.

La méthode présentée ici est une extension de la méthode de Wigner-Seitz [10]. Un traitement analogue a déjà été utilisé par Roth [11]. Une démonstration simple en est présentée ici. Elle est basée essentiellement sur le fait que les surfaces d'énergie constante sont sphériques.

Soit

$$
\Psi_{k}=\mathrm{e}^{i \mathbf{r} . \mathbf{k}} u(\boldsymbol{r}, \boldsymbol{k})
$$

la fonction de Bloch de vecteur d'onde $\boldsymbol{k}$, où $u(\boldsymbol{r}, \boldsymbol{k})$ est périodique. L'équation donnant $u(\boldsymbol{r}, \boldsymbol{k})$ est :

$$
\begin{aligned}
\{\Delta+2[E(\boldsymbol{k})-V(\boldsymbol{r})]\} & u(\boldsymbol{r}, \boldsymbol{k}) \\
+ & 2 i \boldsymbol{\nabla} u(\boldsymbol{r}, \boldsymbol{k}) \cdot \boldsymbol{k}-u(\boldsymbol{r}, \boldsymbol{k}) k^{2}=0 .
\end{aligned}
$$

En développant $\mu(\boldsymbol{r}, \boldsymbol{k})$ et $E(\boldsymbol{k})$ en série de $\boldsymbol{k}$, on cherche de manière générale les solutions de l'équation de Schrödinger d'énergie $E$ sous la forme :

$$
\Psi=u(\boldsymbol{r},-i \boldsymbol{\nabla}) \chi(\boldsymbol{r})
$$

où $\boldsymbol{\nabla}$ n'opère qu'à droite. On obtient ainsi :

$$
u(\boldsymbol{r},-i \boldsymbol{\nabla})[E(-i \boldsymbol{\nabla})-E] \chi(\boldsymbol{r})=0 .
$$

Dans l'approximation de Wigner-Seitz, $E$ ne dépend que de $k^{2}$ et dans chaque cellule assimilée à une sphère, $u(\boldsymbol{r}, \boldsymbol{k})$ a la symétrie de révolution autour de $\boldsymbol{k}$; alors les solutions de

$$
[E(-\Delta)-E] \chi=0
$$

vérifient l'équation (2).

Si $k$ est tel que $E\left(k^{2}\right)=E$, les solutions de

$$
\left(\Delta+k^{2}\right) \chi=0
$$

sont solutions de (2).

En particulier, elles peuvent prendre la forme :

$$
\chi_{l}^{m}=u(\boldsymbol{r},-i \boldsymbol{\nabla}) Y_{l}^{m}(\Omega) j_{l}(k r)
$$

où $Y_{l}^{m}$ est l'harmonique sphérique usuelle et $j_{l}(k r)$ la fonction de Bessel sphérique régulière à l'origine. En prenant l'origine au centre d'une cellule atomique, les solutions (4) ont la symétrie sphérique $(l m)$ à l'intérieur de la sphère centrale : en effet, dans l'expression de $\bar{u}(r,-i \boldsymbol{\nabla}), \boldsymbol{\nabla}$ n'intervient que sous la forme $\boldsymbol{r} \cdot \boldsymbol{\nabla}$.

Si on introduit une impureté dans la cellule cen-

(1) On pourrait se demander s'il n'y a pas un effet similaire dans le cas du Knight shift des alliages dilués. En fait, il n'en est rien (cf. appendice). 
trale, créant un potentiel perturbateur $V_{\mathbf{P}}(r)$, l'équation (2) est transformée en :

$$
\begin{aligned}
u(\boldsymbol{r},-i \boldsymbol{\nabla})[E(-i \boldsymbol{\nabla}) & -E] \varphi(\boldsymbol{r}) \\
& +V_{\mathbf{P}}(\boldsymbol{r}) u(\boldsymbol{r},-i \boldsymbol{\nabla}) \varphi(\boldsymbol{r})=0 .
\end{aligned}
$$

En définissant l'opérateur $W_{\mathbf{P}}(\boldsymbol{r},-i \boldsymbol{\Delta})$ par :

$$
u(\boldsymbol{r},-i \boldsymbol{\nabla}) W_{\mathrm{P}}(r,-i \boldsymbol{\nabla})=V_{\mathbf{P}}(r) u(\boldsymbol{r},-i \boldsymbol{\nabla}),
$$

l'équation (5) est remplacée par :

$u(\boldsymbol{r},-i \boldsymbol{\nabla})\left[E(-i \boldsymbol{\nabla})-E+W_{\mathbf{P}}(\boldsymbol{r},-i \boldsymbol{\nabla})\right] \varphi(\boldsymbol{r})=0$.

Dans l'approximation de Wigner-Seitz et avec un potentiel $V_{\mathbf{P}}(\boldsymbol{r})$ sphérique et nul à l'extérieur de la sphère de Wigner-Seitz, $u(r,-i \nabla)$ et $W_{\mathbf{P}}(\boldsymbol{r},-i \nabla)$ ne dépendent que de $r$ et $\partial / \partial r$ et l'équation (3) devient :

$$
\left[E(-\Delta)-E+W_{\mathbf{P}}\left(r, \frac{\partial}{\partial r}\right)\right] \varphi=0 .
$$

On peut analyser les solutions d'énergie donnée en fonctions de symétrie $(l m)$ à l'intérieur de la sphère :

$$
\varphi_{l}^{m}=Y_{l}^{m}(\Omega) f_{l}(r)
$$

à l'extérieur de la sphère, elles seront de la forme : $\varphi_{l}^{m}=u(\boldsymbol{r},-i \boldsymbol{\nabla}) Y_{l}^{m}(\Omega)\left[\cos \delta_{l} j_{l}(k r)-\sin \delta_{l} n_{l}(k r)\right]$.

Les quantités $\delta_{l}$ sont exactement les analogues des déphasages dans la théorie de la diffusion de pariicules non soumises à un potentiel périodique. On- peut les calculer soit en ajustant les fonctions $\varphi_{l}^{m}$ et leurs dérivées à la surface de la sphère de Wigner-Seitz, soit en résolvant l'équation (6) : si l'on se borne, dans le développement de $E(k)$ et de $u(\boldsymbol{r}, \boldsymbol{k})$ aux termes du second ordre en $\boldsymbol{k}$, l'équation (6) devient

$$
\left\{\Delta+2 m^{*}\left[E-W_{\mathbf{P}}\left(r, \frac{\partial}{\partial r}\right)\right]\right\} \varphi=0 .
$$

où $m^{*}$ est la masse effective de bas de bande. $\mathrm{Si}$

$$
\begin{aligned}
u(\boldsymbol{r}, \boldsymbol{k})=u_{0}(\boldsymbol{r})+i \boldsymbol{k} \cdot \frac{\boldsymbol{r}}{r} u_{0}(\boldsymbol{r})+ & \ldots, \\
W_{\mathbf{P}}\left(r, \frac{\partial}{\partial r}\right)=V_{\mathbf{P}}(r) & -\frac{u_{0}(r)}{u_{0}(r)} \frac{\mathrm{d} V_{\mathbf{P}}}{\mathrm{d} r} \\
& +\left[\frac{u_{1}(r)}{u_{0}(r)}\right]^{2} \frac{\mathrm{d} V_{\mathbf{P}}}{\mathrm{d} r} \frac{\partial}{\partial r}+\ldots
\end{aligned}
$$

L'équation (7) justifie l'emploi d'électrons libres, en particulier dans les calculs de résistivité des alliages $[4,12]$.

V. Variations de densité électronique et gradients de champ. - De la forme (6) des fonctions d'onde se déduit la variation totale de densité électronique $\Delta \rho_{\mathbf{A}}(\boldsymbol{r})$ au point $\boldsymbol{r}$ pour grand $\boldsymbol{r}$.
Le terme principal pour grand $r$ de $\varphi_{l}^{m}$ est :

$$
\begin{aligned}
\varphi_{l}^{m}=u(\boldsymbol{r},- & i \boldsymbol{\nabla}) \frac{Y_{l}^{m}(\Omega)}{k r} \sin \left(k r+\delta_{l}-\frac{l \pi}{2}\right) \\
= & \frac{Y_{l}^{m}(\Omega)}{k r}\left[\left[u(\boldsymbol{r}, \boldsymbol{K}) \mathrm{e}^{i\left(k r+\delta_{l}-\frac{l \pi}{2}\right)}\right.\right. \\
& \left.\quad-u(\boldsymbol{r},-\boldsymbol{K}) \mathrm{e}^{-i\left(k r+\delta_{l}-\frac{l \pi}{2}\right)}\right]
\end{aligned}
$$

où $\boldsymbol{K}=k \frac{\boldsymbol{r}}{\boldsymbol{r}}$ et les fonctions $u(\boldsymbol{r}, \boldsymbol{K})$ sont normalisées à l'unité dans l'unité de volume.

On en tire, en utilisant le fait que

$$
\begin{gathered}
u^{*}(\boldsymbol{K})=u(-\boldsymbol{K}): \\
\left|\varphi_{l}^{m}\right|^{2}-\left|\chi_{l}^{m}\right|^{2}=(-1)^{l+1} \frac{\left|Y_{l}^{m}(\Omega)\right|^{2}}{4 k^{2} r^{2}} \\
\left\{[u(\boldsymbol{r}, \boldsymbol{K})]^{2} \mathrm{e}^{i 2 k r}\left(\mathrm{e}^{2 i \delta_{l}}-1\right)+c c\right\} .
\end{gathered}
$$

Il vient alors pour $\Delta \rho_{\mathbf{A}}(\boldsymbol{r})$ :

$$
\begin{aligned}
& \Delta \rho_{\mathbf{A}}(\boldsymbol{r})=\sum_{l}(-1)^{l} \frac{(2 l+1)}{2 i \pi^{2} r^{2}} \\
& \int_{0}^{k} \mathrm{~F} \sin \delta_{l}\left\{\left[u(\boldsymbol{r}, \boldsymbol{K})^{2} \mathrm{e}^{i\left(2 k r+\delta_{l}\right)}-c c\right\} \mathrm{d} k\right.
\end{aligned}
$$

et en prenant le terme principal pour grand $r$, par intégration par parties :

$$
\begin{aligned}
\Delta \rho_{\mathbf{A}}(\boldsymbol{r})=-\sum_{\boldsymbol{i}}-(-1) l & \frac{(2 l+1) \sin \delta_{l}^{F}}{4 \pi^{2} r^{3}} \\
& \left\{\left[u\left(\boldsymbol{r}, \boldsymbol{K}_{\mathrm{F}}\right)\right]^{2} \mathrm{e}^{i\left(2 k_{\mathrm{F}} r+\delta_{l}^{F}\right)}+c c\right\}
\end{aligned}
$$

où $[u(\boldsymbol{K})]^{2}$ signifie fonction $u$ au carré et non module au carré; $\dot{\boldsymbol{K}}_{\mathrm{F}}=k_{\mathrm{F}} \frac{\boldsymbol{r}}{r}, k_{\mathrm{F}}$ et $\delta_{l}^{\mathbf{F}}$ sont le vecteur d'onde et le déphasage $l$ au niveau de Fermi. Pour des électrons libres, le résultat serait simplement :

$$
\Delta \rho_{\mathbb{A}}^{F}=-\sum_{l}(-1)^{l} \frac{(2 l+1) \sin \delta_{l}^{F}}{2 \pi^{2} r^{3}} \cos \left(2 k_{\mathrm{F}}+\delta_{l}^{F}\right),
$$

résultat qui peut s'établir directement (Friedel [4]) et se mettre sous la forme :

$$
\Delta \rho_{\mathbf{A}}^{F}=-\frac{\alpha}{2 \pi^{2} r^{3}} \cos \left[2 k_{\mathrm{F}} r+\varphi\right]
$$

$\alpha$ et $\varphi$ étant déterminés par :

$$
\left\{\begin{array}{l}
\alpha \cos \varphi=\frac{1}{2} \sum_{l}(-1)^{l}(2 l+1) \overline{\sin } 2 \delta_{l}^{F} \\
\alpha \sin \varphi=\sum_{l}(-1)^{l}(2 l+1) \cdot \sin ^{2} \delta_{l}^{F} .
\end{array}\right.
$$

Les oscillations de densité électronique données par (8) ou (9) ne dépendent que des fonctions d'onde et de la diffusion au niveau de Fermi. Comme elles sont obtenues dans l'approximation à un élec- 
tron, on peut se demander quelle est leur validité et si l'interaction coulombienne entre électrons ne modifie pas profondément ces résultats. On peut montrer qu'il n'en est rien et qu'à grande distance, on trouve des résultats analogues en tenant compte de l'interaction.

Une autre question est de savoir l'erreur commise en confondant la valeur asymptotique pour grand $r$ avec la valeur exacte. En fait, on peut vérifier que, même pour la distance $r$ correspondant aux premiers voisins, la formule (9) est assez bonne, dans le cas d'électrons libres. On peut donc raisonnablement espérer qu'il en est de même dans le cas des électrons de Bloch.

$\Delta \rho(\boldsymbol{R})$ étant la variation de densité électronique en $\boldsymbol{R}, Z$ la charge de l'impureté écrantée par la répartition $\Delta \rho(\boldsymbol{R})$, le tenseur de champ gradient en $\boldsymbol{R}_{\mathbf{i}}$ créé par les champs extérieurs au noyau est alors :

$$
\overline{\overline{G\left(\mathbf{R}_{\mathbf{i}}\right)}}=-\int[\Delta \rho(\mathbf{R})-Z \delta(\mathbf{R})] \overline{\overline{\frac{1}{\left|\mathbf{R}-\mathbf{R}_{\mathbf{i}}\right|}}} d^{3} \mathbf{R} .
$$

En supposant que la répartition de charges a la symétrie de révolution autour de $\boldsymbol{R}_{\mathbf{i}}$ (ce qui est vrai en moyenne à la limite des grands $\boldsymbol{R}_{\mathrm{i}}$ ), on obtient pour la composante suivant $\boldsymbol{R}_{\mathrm{i}}$ :

$G\left(R_{\mathrm{i}}\right)=-2 \int[\Delta \rho(\mathbf{R})-Z \delta(\mathbf{R})] \frac{P_{2}(\cos \theta)}{r^{3}} \mathrm{~d}^{3} \boldsymbol{r}$

où $P_{2}$ est le polynôme de Legendre d'ordre $2, r$ et $\theta$ ayant la signification donnée sur la figure 1.

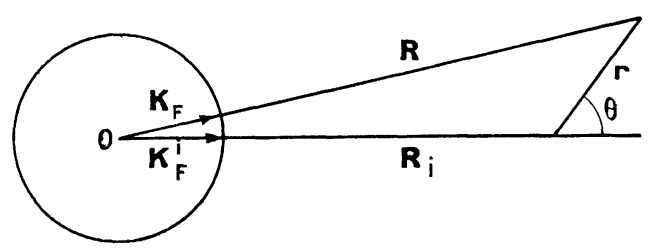

Fig. 1.

La plus grande partie de la charge est située dans la cellule de Wigner-Seitz entourant l'impureté et possède la symétrie sphérique dans l'approximation de Wigner-Seitz utilisée ici. Pour calculer $G\left(\boldsymbol{R}_{\mathrm{i}}\right)$, on peut donc procéder comme suit : comme la charge $Z$ écrantée est pratiquement concentrée dans la cellule centrale, on considère une sphère (S) ayant approximativement les dimensions de la sphère de Wigner-Seitz et contenant exactement une charge :

$$
\int_{R \in(\mathbb{S})} \Delta \rho(\mathbf{R}) d^{3} \mathbf{R}=Z .
$$

L'ensemble de cette charge et de la charge $Z$ à écranter donnent un gradient de champ nul à grande distance (à l'ordre $\left.1 / R_{\mathrm{i}}^{z}\right)$. Il suffit alors d'évaluer l'influence de $\Delta \rho(\boldsymbol{R})$ à l'extérieur de la sphère : on peut alors confondre $\Delta \rho(\boldsymbol{R})$ avec sa forme asymptotique $\Delta_{\boldsymbol{\Lambda}} \rho(\boldsymbol{R})$ :

$$
G\left(R_{\mathbf{i}}\right)=-2 \int \Delta \rho_{\mathrm{A}}(\mathbf{R}) \frac{P_{\mathbf{2}}(\cos \theta)}{r^{3}} \mathrm{~d}^{3} \boldsymbol{r} .
$$

La contribution importante vient des charges à proximité du noyau $\boldsymbol{R}_{\mathbf{i}}$ et on limite l'intégrale donnant $G\left(R_{\mathrm{i}}\right)$ à la sphère de Wigner-Seitz entourant $\boldsymbol{R}_{\mathbf{i}}\left({ }^{1}\right)$. A l'ordre $1 / R_{\mathbf{1}}^{\mathbf{3}}$, on peut négliger la variation de $R$ dans le terme en $1 / R_{1}^{\mathbf{3}}$ de $\Delta_{\rho_{F}}(\boldsymbol{r})$ et confondre $\boldsymbol{K}_{\mathbf{F}}$ avec $\boldsymbol{K}_{\mathbf{i}}^{\mathbf{F}}=k_{\mathbf{F}} \boldsymbol{R}_{\mathbf{i}} / R_{\mathbf{i}}$. On obtient alors :

$$
G\left(R_{\mathbf{i}}\right)=A \frac{\cos \left(2 k_{\mathbf{F}} R_{\mathbf{i}}+\varphi\right)}{R_{\mathbf{i}}^{\mathbf{3}}}
$$

avec :

$$
A=\frac{8 \pi}{3} \frac{\alpha}{2 \pi^{2}} \mu
$$

et

$$
\mu=\frac{3}{8 \pi} \int_{\text {W.S. }} \mathrm{e}^{2 i \mathbf{K}_{\mathbf{F}}^{\frac{1}{1}} \cdot \mathbf{r}}\left[u\left(\boldsymbol{r}, \boldsymbol{K}_{\mathbf{F}}^{\mathbf{i}}\right)\right]^{2} \frac{P_{2}(\cos \theta)}{r^{3}} \mathrm{~d}^{3} \boldsymbol{r},
$$

$\alpha$ et $\varphi$ sont définis par (9).

Le cas d'électrons libres donnerait exactement $\mu=1$ ainsi qu'un calcul direct peut le montrer. $\mu$ représente donc l'influence de la structure de bandes au niveau de Fermi du métal pur.

VI. Évaluation des gradients de champ. - Le calcul numérique des gradients de champ nécessite la connaissance des constantes $\alpha, \varphi$ et $\mu$ :

$\alpha$ et $\varphi$ ne dépendent que des déphasages au niveau de Fermi ; ils peuvent en principe être calculés en connaissant le potentiel et raccordent les fonctions d'onde à la surface de la sphère de Wigner-Seitz entourant l'impureté. Mais la connaissance exacte du potentiel de l'impureté n'est pas essentielle : en effet, pratiquement seuls les déphasages $s$ et $p$ sont appréciables et on impose d'autre part deux conditions, la règle de Friedel [4]:

$$
Z=\frac{2}{\pi} \sum_{l}(2 l+1) \delta_{l}^{\mathrm{F}}
$$

qui garde sa validité dans l'approximation utilisée [11] et la portée moyenne du potentiel. On peut donc utiliser un potentiel assez grossier et obtenir des résultats acceptables.

Les déphasages utilisés dans le cas des alliages de cuivre ont été calculés par Blatt [12] dans l'approximation des électrons libres et avec un potentiel en puits carré de rayon $r_{\mathrm{s}}$, en tenant compte de l'effet de taille : cela revient à remplacer dans la règle de Friedel la différence de valence $Z$ entre l'impureté et la matrice par

$$
Z_{\text {eff }}=Z+\frac{3 a-r_{\mathbf{A}}}{r_{\mathbf{A}}} z,
$$

(1) Ceci n'est vrai que pour des densités électroniques assez fortement concentrées sur les noyaux (ce qui est le cas pour les métaux considérés plus loin). L'appendice B traite le même problème avec des électrons libres: les gradients sont alors beaucoup plus faibles. 
où $z$ est la valence de la matrice. Le tableau I donne les valeurs de $\alpha$ et $\varphi$ ainsi calculées pour les alliages de cuivre. Il est à noter la forte variation de $\alpha$ avec $Z_{\text {eff }}$ qui devrait pouvoir être observée expérimentalement.

\section{TABLEAU I}

Valeurs des constantes $\alpha$ et $\varphi$ dans les alliages de cuivre

$\begin{array}{ccccc}\text { Impureté } & \mathrm{Zn} & \mathrm{Ga} & \mathrm{Ge} & \mathrm{As} \\ - & - & - & - & - \\ \alpha & 0,28 & 0,65 & 1,3 & 1,74 \\ \varphi \text { en radians } & 1,87 & 3,67 & 4,31 & 4,88 \\ Z_{\text {eff }} & 0,89 & 1,84 & 2,81 & 3,73\end{array}$

Pour les alliages d'aluminium n'ayant pas les valeurs calculées de déphasages, on a simplement ajusté les valeurs de $\delta_{0}$ et $\delta_{1}$ de manière à satisfaire la règle de Friedel et obtenir la résistivité mesurée [13] qui, dans l'approximation de WignerSeitz, est donnée par la même formule que pour des électrons libres [14]:

$$
\Delta=\frac{4 \pi \hbar c}{z k_{\mathrm{F}} e^{2}} \sum_{l} l \sin ^{2}\left(\delta_{l}^{\mathbf{F}}-\delta_{i-1}^{\mathbf{F}}\right) .
$$

Cette méthode doit être assez bonne car le rayon d'écran dans l'aluminium est plus petit que celui du cuivre et l'influence des déphasages $d$, déjà peu importante dans le cas du cuivre, doit être négligeable. Le tableau II donne les valeurs trouvées.

\section{TABLEAU II}

Valeurs de $\delta_{0}, \delta_{1}, \alpha$ et $\varphi$ dans les alliages $\mathbf{A l} \mathrm{Zn}$ et $\mathbf{A l} \mathrm{Mg}$

( $\Delta$ en $\mu \Omega$ cm par at. \%)

$$
\begin{array}{ccccccc} 
& Z_{\text {eff }} & \Delta & \delta_{\mathbf{0}} & \delta & \alpha & \varphi \\
\mathrm{Zn} & -\overline{0,90} & 0,35 & -0,715 & -0,228 & 0,32 & 2,11 \\
\mathrm{Mg} & -1,68 & 0,60 & -2,403 & -0,078 & 0,51 & 2,12
\end{array}
$$

Le coeffic ent $\mu$ dépend des fonctions d'onde au niveau de Fermi du métal pur. C'est une constante pour chaque série d'alliages. Un rapide calcul numérique montre que les effets observés dans l'aluminium et le cuivre ne peuvent 'être expliqués si l'on ne tient compte que de la partie $s$ des fonctions périodiques $u\left(\boldsymbol{r}, \boldsymbol{k}_{\mathrm{F}}\right)$. (Dans le cas du cuivre, on obtiendrait une valeur de $\mu$ voisine de l'unité.) $\mu$ dépend très fortement du caractère $\left(^{1}\right) p$ et $d$ des fonctions d'onde.

(1) Les caractères $s, p, d \ldots$ des fonctions $u(\boldsymbol{r}, \boldsymbol{K})$ est ainsi défini. Soit :

$$
u(\boldsymbol{r}, \boldsymbol{K})=a_{\mathrm{B}} u_{\mathrm{g}}(\boldsymbol{r})+a_{\mathrm{p}} u_{\mathrm{p}}(\boldsymbol{r})+a_{\mathrm{d}} u_{\mathrm{d}}(\boldsymbol{r}) \text { etc... } ;
$$

les fonctions $u\left(\boldsymbol{r}, \boldsymbol{K} u_{\mathrm{s}}(\boldsymbol{r})\right.$... étant normalisées à l'unité dans l'unité de volume, on a :

$$
1=\left|a_{\mathrm{B}}\right|^{2}+\left|a_{\mathrm{p}}\right|^{2}+\cdots
$$

$\left|a_{\mathrm{B}}\right|^{2}$ est la mesure du caractère $s$.
Dans le cas de l'aluminium, le caractère $p$ est important ainsi que le montrent les calculs de Heine [15]. Un calcul par la méthode des ondes planes orthogonalisées donne $\mu_{\mathbf{A}_{1}} \simeq 5$ (voir Appendice $\mathrm{C}$ ). En fait, pour expliquer les résultats expérimentaux, une valeur de $\mu_{A_{1}}$ égale à 15 est nécessaire. Cette différence n'est pas étonnante car le calcul par ondes planes orthogonalisées tient mal compte du fait que certaines régions de la surface de Fermi pour lesquelles $\nabla E(k)$ est petit ont un caractère $p$ très marqué. Un calcul plus détaillé serait nécessaire, en sortant de l'approximation de WignerSeitz. Mais alors le calcul de la diffusion devient délicat.

Dans le cas du cuivre, les caractères $p$ et $d$ doivent être importants. En tenant compte uniquement de la partie $d$ des fonctions d'onde, il faudrait un caractère $d$ de l'ordre de 0,1 pour rendre compte de la valeur déduite de l'expérience $\mu_{\mathrm{cu}} \simeq 30$.

VII. Comparaison aux résultats expérimentaux. - Aveo la valeur $\mu_{\mathbf{A 1}_{1}}=15$, les gradients de champ pour les voisins d'un atome de zinc ou de magnésium sont donnés dans le tableau III. La limite $\boldsymbol{q}_{\mathbf{0}}$

\section{TABLEAU III}

Valeurs des gradients de champ (en $10^{21} \mathrm{~cm}^{-3}$ ) dans les alliages $\mathrm{Al} \mathrm{Zn}$ et $\mathrm{Al} \mathrm{Mg}$

$$
\begin{aligned}
& \begin{array}{lllllllllll}
\text { Pts } & 011 & 022 & 112 & 022 & 013 & 222 & 123 & 004 & 114 & 024
\end{array} \\
& \begin{array}{ccccccccccccc}
- & - & - & - & - & - & - & - & - & - & - \\
\text { Nombre } & 12 & 6 & 24 & 12 & 24 & 8 & 48 & 6 & 24 & 24
\end{array} \\
& q\left\{\begin{array}{lllllllllll}
\mathrm{Zn} & 78 & 26 & 9,3 & 10,8 & 4,7 & 0,6 & 4,9 & 2,0 & 2,1 & 1,9
\end{array}\right. \\
& q\left\{\begin{array}{lllllllllll}
\mathrm{Mg} & 126 & 42 & 15 & 17 & 7,5 & 1 & 7,2 & 3,3 & 3,3 & 3
\end{array}\right.
\end{aligned}
$$

a été choisie égale à $6,10^{21} \mathrm{~cm}^{-3}$ ainsi que Rowland [2] le suppose. On obtient en particulier des gradients pour les premiers voisins égaux à 78 et $125.10^{21} \mathrm{~cm}^{-3}$. Rowland donne la valeur, limite de $120.10^{21} \mathrm{~cm}^{-3}$ pour observer une déformation de la raie de résonance par effet du second ordre. Cet effet est effectivement observé dans le cas $\mathbf{A l ~ M g}$, mais non dans le cas $\mathbf{A l} \mathbf{Z n}$. L'ensemble de ces résultats en particulier la variation du nombre $n$ de 98 à 130 pour $\mathrm{Zn}$ et $\mathrm{Mg}$ est bien expliquée par le modèle proposé.

Dans le cas des alliages $\mathbf{C u} \mathrm{Zn}$, il est nécessaire d'avoir des gradients de $150.10^{21} \mathrm{~cm}^{-3}$ aux sites des premiers et seconds voisins. Avec $\mu_{\mathrm{cu}}=30$, on obtient pour les premiers, deuxièmes et troisièmes voisins les valeurs de $q: 225,155$ et $25.10^{21} \mathrm{~cm}^{-3}$.

En conclusion on peut dire que les résultats expérimentaux sont qualitativement bien compris par la théorie ci-dessus. La principale imprécision vient de la valeur numérique du coefficient $\mu$, qui 
est estimé assez grossièrement à 5 pour l'aluminium. Mais les valeurs de 15 et 30 pour $\mathrm{Al}$ et $\mathrm{Cu}$ sont raisonnables et on peut penser qu'un calcul détaillé des fonctions d'onde au niveau de Fermi donnerait des résultats voisins de ces nombres.

Deux faits essentiels caractérisent cette théorie : d'une part l'influence de la structure des bandes est d'amplifier fortement les effets qui seraient obtenus avec des électrons libres. Un effet analogue est sans doute la cause des très fortes réductions d'intensité de résonance nucléaire observées dans les semiconducteurs InSb et GaSb [16] contenant de petites concentrations d'impuretés. D'autre part, l'existence d'interactions à grande distance dans les métaux (7,5 $\AA$ pour le cas $\mathbf{A l ~ M g ) ~ e s t ~ s o u l i g n e ́ e . ~}$ Ce fait est à rapprocher des interactions entre impuretés magnétiques dans les alliages dilués Cu Mn [6]. Dans les deux cas, les effets à grande distance ne dépendent que de la diffusion au niveau de Fermi.

Appendice A : Sur le déplacement de Knight. Aux sites d'un noyau. $u(\boldsymbol{r}, \boldsymbol{k})$ ne dépend de $\boldsymbol{k}$ que sous la forme $\boldsymbol{k}^{2} ;$ en effet, dans $u(\boldsymbol{r}, \boldsymbol{k}), \boldsymbol{k}$ apparait sous la forme $k^{2}$ ou (k.r) multiplié par une fonction finie pour $\boldsymbol{r}=0$. Ce résultat est d'ailleurs évident car, dans l'approximation de Wigner-Seitz, il n'y a aucune direction privilégiée.

Les fonctions $\chi_{l}^{m}\left(R_{\mathrm{i}}\right)$ et $\varphi_{l}^{m}\left(R_{\mathrm{i}}\right)$ sont donc, en dehors de la sphère centrale :

$\chi_{l}^{m}\left(\boldsymbol{R}_{\mathbf{i}}\right)=Y_{l}^{m}(\Omega) j_{l}\left(k, R_{\mathbf{i}}\right) u\left(\boldsymbol{R}_{\mathbf{i}}, \boldsymbol{k}^{2}\right)$

$\varphi_{l}^{m}\left(R_{\mathbf{i}}\right)=Y_{l}^{m}(\Omega)\left[\cos \delta_{l} j_{l}\left(k, R_{\mathbf{i}}\right)-\sin \delta_{l} n_{l}\left(k, R_{\mathrm{i}}\right)\right] u\left(\boldsymbol{R}_{\mathbf{i}}, k^{2}\right)$

et le changement relatif de densité au point $r$, et au niveau de Fermi est :

$$
\begin{aligned}
\frac{\Delta \rho(\boldsymbol{r})}{\rho(r)}=\sum_{l}(2 l+1) & \left\{\left[n_{l}^{2}\left(k_{\mathrm{F}} r\right)-j_{l}^{2}\left(k_{\mathrm{F}} r\right)\right] \sin ^{2} \delta_{l}^{\boldsymbol{F}}\right. \\
& \left.-j_{l}\left(k_{\mathrm{F}} r\right) n_{l}\left(k_{\mathrm{F}} r\right) \sin 2 \delta^{\mathbf{F}}\right\} .
\end{aligned}
$$

C'est exactement la même formule que dans le cas d'électrons libres [5].

Appendice B : Calcul du gradient de champ pour des électrons libres. - Si les électrons ne sont pas soumis à un potentiel périodique, la répartition de charge électronique $\Delta \rho^{\mathrm{F}}(r)$ autour de l'impureté à la symétrie sphérique. L ${ }^{\circ}$ potentiel en $R$ est alors :

$$
\begin{gathered}
V(R)=\frac{Z}{R}-\frac{1}{R} \int_{0}^{R} \Delta \rho \mathrm{F}(r) 4 \pi r^{2} d r-\int_{R}^{+\infty} \Delta \rho \mathrm{F}(r) 4 \pi r \mathrm{~d} r \\
=\frac{1}{R} \int_{R}^{+\infty} \Delta \rho \mathrm{F}(r) 4 \pi r^{2} \mathrm{~d} r-\int_{R}^{+\infty} \Delta \rho^{\mathrm{F}}(r) 4 \pi r \mathrm{~d} r .
\end{gathered}
$$

(On a utilisé le fait que $\Delta \rho^{F}(r)$ sommé sur tout l'espace est égal à $Z$.)

La composante radiale du champ est :

$$
E(R)=-\frac{\partial V}{\partial R}=\frac{1}{R^{2}} \int_{R}^{+\infty} \Delta \rho \mathrm{F}(r) 4 \pi r^{2} \mathrm{~d} r,
$$

d'où le gradient :

$$
G(R)=\frac{\partial E}{\partial R}=-\frac{2}{R^{3}} \int_{R}^{+\infty} \Delta \rho \mathrm{F}(r) \mathrm{d} \pi r^{2} \mathrm{~d} r-4 \pi \rho(R)
$$

à grande distance de l'impureté :

$$
\Delta \rho \mathbf{F}(r)=-\frac{\alpha}{2 \pi^{2}} \frac{\cos \left(2 k_{\mathrm{F}} r+\varphi\right)}{r^{3}}
$$

et

$$
G(R)=4 \pi \frac{\alpha}{2 \pi^{2}} \frac{\cos \left(2 k_{\mathrm{F}} R+\varphi\right)}{R^{3}} .
$$

Le gradient $G(R)$ ainsi calculé est le gradient total créé par les charges extérieures et sur le noyau; le gradient $G_{0}(R)$ créé par les charges extérieures est donc :

$$
\begin{aligned}
G_{0}(R) & =G(R)+\frac{4 \pi}{3} \rho(R) \\
& =\frac{8 \pi}{3} \frac{\alpha}{2 \pi^{2}} \frac{\cos \left(2 k_{\mathrm{F}} R+\varphi\right)}{R^{3}}
\end{aligned}
$$

Dans le cas d'électrons libres, le coefficient $\mu$ est donc égal à 1 .

Appendice C : Calcul de $\mu_{\mathrm{A}_{1}}$ - - Dans l'approximation des bandes sphériques, on prend comme fonctions d'onde approchées les fonctions :

$$
\Psi_{\mathbf{k}}(\boldsymbol{r})=\alpha\left[\mathrm{e}^{i \mathbf{k r}}-a_{1 s} \Psi_{1 s}-a_{2 s} \Psi_{2 s}-a_{2 p} \Psi_{2 p}\right]
$$

où $\Psi_{1 s}, \Psi_{2 s}, \Psi_{2^{p}}$ sont les fonctions d'onde atomiques de l'ion $\mathrm{Al}^{+++}$calculées par Hartree [17]. Les coefficients $a_{1 s}, a_{2 s}$ et $a_{2 p}$ sont déterminés de façon que $\Psi_{\mathbf{k}}(\boldsymbol{r})$ soit orthogonale aux fonctions atomiques internes. $\alpha$ est un coefficient normalisant la fonction $\Psi_{\mathbf{k}}(\boldsymbol{r})$ à l'unité dans l'unité de volume.

Dans le système d'unités atomiques, les valeurs des coefficients pour $k=k_{\mathrm{F}}$ sont :

$$
a_{1 s}=0,32 \quad a_{2 s}=3,25 \quad a_{2 p}=1,61 i
$$

et

$$
\alpha=1,06 .
$$

En analysant $\left[\Psi_{\mathbf{k}}(\boldsymbol{r})\right]^{2}$ en harmoniques sphériques, et en reportant dans (13), on en tire :

$$
\mu_{A 1} \simeq 5 .
$$

Manuscrit reçu le 4 mai 1960. 


\section{RÉFÉRENCES}

[1] Bloembergen (N.) et Rowland (T. J.), Acta Met., 1953, 1, 731 .

[2] Rowland (T. J.), Acta Met., 1955, 3, 74.

[3] Bloembergen (N.), Report Conf. on Defects in Crystalline Solids, 1954, p. 1 ; Physical Society, London, 1955.

[4] Friedel (J.), Phil. Mag., 1952, 43, 153. Cours de l'École de Varenna, 1957, Suppl. Nuovo Cim., 1958, $2,287$.

[5] Blandin (A.), Daniel (E.) et Friedel (J.), Phil. Mag., 1959, 4, 180.'Blandin (A.) et Daniel (E.), J. Phys. Chem. Sol., 1959, 10, 126.

[6] Blandin (A.) et Friedel (J.), Colloque Intern. Magnétis̀me, Grenoble, 1958 ;J. Physique Rad., 1959, 20, 160.

[7] Friedel (J.), Les Dislocations, Gauthier-Villars, Paris, 1956.
[8] Smithells (C. J.), Meials Reference Book, Butterworths Scientific Publications, London, 1955.

[9] Averbuch (P.), De Bergevin (F.) et MullerWarmuth (W.), C.R.A.S., 1959, 249, 2315.

[10] Wigner (E. P.) et Seitz (F.), Phys. Rev., 1934, 46, 509.

[11] Rотн (L. M.), Thèse, Harvard University, 1957.

[12] Blatt (F. J.), Phys. Rev., 1957, 108, 285.

[13] Vassel (C. R.), J. Phys. Chem. Sol., 1958, 7, 90 ; Bergeakad., 1957, 6, 306.

[14] Huang (K.), Proc. Phys. Soc., 1948, 60, 161.

[15] Heine (V.), Proc. Phys. Soc., 1957, 240 A, 340.

[16] Rhoderick (E. H.), Phil. Mag., 1958, 3, 545. Oliver (D. J.), J. Phys. Chem. Sol., 1959, 11, 257.

[17] Hartree (D.), Proc. Roy. Soc., 1935, A 151, 96.

[18] Koнn (W.) et Vosko (S. H.), à paraître. 